\title{
A Mathematical Framework for Structural Control Integration
}

\author{
Josep M. Rossell ${ }^{1, a}$, José Rodellar²,b , Francisco Palacios-Quiñonero ${ }^{1, \mathrm{c}}$ \\ and Josep Rubió-Massegú1,d \\ ${ }^{1}$ Department of Applied Mathematics III, Universitat Politècnica de Catalunya (UPC). \\ Av. Bases de Manresa, 61-73, 08242-Manresa, Barcelona, Spain. \\ ${ }^{2}$ Department of Applied Mathematics III, Universitat Politècnica de Catalunya (UPC). \\ Campus Nord, Edifici C2, 08034-Barcelona, Spain. \\ ${ }^{a}$ josep.maria.rossell@upc.edu, ${ }_{d}^{b}$ jose.rodellar@upc.edu, ${ }^{c}$ francisco.palacios@upc.edu,
}

Keywords: Structural vibration control, decentralized control, overlapping decomposition, inclusion principle.

\begin{abstract}
In this paper, some control strategies to design decentralized controllers are developed and discussed. These strategies are based on the Inclusion Principle, a very useful mathematical framework to obtain decentralized controllers, mainly when the systems are composed by overlapped subsystems sharing common parts. A five-story building model serves as example to show the advantages provided by this approach. Numerical simulations are conducted to assess the performance of the proposed control laws with positive results.
\end{abstract}

\section{Introduction}

Vibration control of flexible structures, in particular tall buildings, under strong winds and/or seismic excitations has attracted considerable attention in recent years. In order to avoid the undesirable effects of vibrations produced by external stimuli, a large variety of control strategies has been developed, including passive, active, hybrid or semiactive damping methods. Passive devices, as the base isolation systems, offer well-known mechanisms to be implemented in the foundation of the buildings. Mass dampers placed on the top or in the middle of the buildings, or the use of viscoelastic dampers, are other passive control possibilities. Unfortunately, these kinds of devices are not able to react properly to different structural change of conditions [1,2]. To overcome this difficulty, active, hybrid and semiactive control systems are frequently designed to attenuate building vibrations, achieving better performance than passive control methods [3, 4, 5, 6, 7, 8].

Tall buildings can be considered as complex systems and, in this case, they can be decomposed into disjoint subsystems. For this class of systems a set of local controllers may be independently obtained to design decentralized controllers. Some advantages in designing and using local controllers are the following: (1) lower-dimension computation is required; (2) minimization of the information exchange; (3) increment of the global robustness; and (4) reduction of the effect of perturbations and failures on communications. In order to design decentralized controllers, a mathematical framework called Inclusion Principle will be used in the paper. The inclusion principle deals with systems composed by overlapped subsystems which, by means of appropriate linear transformations, can be treated as disjoint. Then, local controllers are designed to be transformed and implemented into the initial systems to control them [9, 10, 11]. This useful approach has been applied in a large variety of complex control problems appearing in different areas, such as electric power generation, automated highway traffic management, civil structural engineering or aerospace structural engineering. 
As a good example of structural control integration, we consider a complex control configuration consisting in several semiactive dampers and sensors installed in different floors of the building together with an appropriate communications system and a suitable feedback control strategy. To improve the robustness of the communications, we suppose that the controllers operate using only local information supplied by neighboring sensors. Consequently, a decentralized control is required for a realistic treatment of wireless networked control systems $[12,13,14]$ and an overlapping approach may be specially convenient $[15,16]$.

In order to present the main ideas, a five-story building model excited by a seismic disturbance has been selected. For this building, three kinds of LQR controllers are designed and compared: (1) a centralized controller, which serves as reference; (2) a semi-decentralized two-overlapping controller; and (3) a semi-decentralized multi-overlapping controller. In all the cases, the El Centro North-South 1940 seismic record has been used as excitation.

\section{The Inclusion Principle}

We summarize some basic definitions and results related to the Inclusion Principle in order to design overlapping controllers. A more detailed treatment can be found in [9, 10, 11].

Consider a pair of linear systems

$$
\mathbf{S}:\left\{\begin{array}{l}
\dot{x}(t)=A x(t)+B u(t) \\
y(t)=C_{y} x(t)
\end{array} \quad \tilde{\mathbf{S}}:\left\{\begin{array}{l}
\dot{\tilde{x}}(t)=\tilde{A} \tilde{x}(t)+\tilde{B} \tilde{u}(t) \\
\tilde{y}(t)=\tilde{C}_{y} \tilde{x}(t)
\end{array}\right.\right.
$$

where $x(t) \in \mathbb{R}^{\mathbf{n}}, u(t) \in \mathbb{R}^{\mathbf{m}}, y(t) \in \mathbb{R}^{1}$ are the state, the input, and the output of $\mathbf{S}$ at time $t \geqslant 0$; $\tilde{x}(t) \in \mathbb{R}^{\tilde{\mathbf{n}}}, \tilde{u}(t) \in \mathbb{R}^{\tilde{\mathbf{m}}}, \tilde{y}(t) \in \mathbb{R}^{\tilde{1}}$ are the state, the input, and the output corresponding to $\tilde{\mathbf{S}} ; A, B$, $C_{y}$ and $\tilde{A}, \tilde{B}, \tilde{C}_{y}$ are $n \times n, n \times m, l \times n$ and $\tilde{n} \times \tilde{n}, \tilde{n} \times \tilde{m}, \tilde{l} \times \tilde{n}$ dimensional matrices, respectively. Let us consider the following linear transformations:

$$
\begin{aligned}
& V: \mathbb{R}^{\mathrm{n}} \longrightarrow \mathbb{R}^{\tilde{\mathbf{n}}}, \quad R: \mathbb{R}^{\mathrm{m}} \longrightarrow \mathbb{R}^{\tilde{\mathbf{m}}}, \quad T: \mathbb{R}^{1} \longrightarrow \mathbb{R}^{\tilde{1}}, \\
& U: \mathbb{R}^{\tilde{\mathbf{n}}} \longrightarrow \mathbb{R}^{\mathbf{n}}, \quad Q: \mathbb{R}^{\tilde{\mathbf{m}}} \longrightarrow \mathbb{R}^{\mathbf{m}}, \quad S: \mathbb{R}^{\tilde{1}} \longrightarrow \mathbb{R}^{1},
\end{aligned}
$$

where $V, R, T$ are called expansion matrices with $\operatorname{rank}(V)=n, \operatorname{rank}(R)=m, \operatorname{rank}(T)=l$, and $U, Q, S$ are contraction matrices obtained by computing $U=\left(V^{T} V\right)^{-1} V^{T}, Q=\left(R^{T} R\right)^{-1} R^{T}$, $S=\left(T^{T} T\right)^{-1} T^{T}$, which satisfy $U V=I_{n}, Q R=I_{m}, S T=I_{l}$, where $I_{n}, I_{m}, I_{l}$ denote the identity matrices of indicated dimensions.

Definition 1. (Inclusion Principle) A system $\tilde{\mathbf{S}}$ includes the system $\mathbf{S}$ if there exists a quadruplet of matrices $(U, V, R, S)$ such that, for any initial state $x_{0}$ and any fixed input $u(t)$ of $\mathbf{S}$, the choice of $\tilde{x}_{0}=V x_{0}, \tilde{u}(t)=R u(t)$ for all $t \geqslant 0$ as initial state $\tilde{x}_{0}$ and input $\tilde{u}(t)$ for the system $\tilde{\mathbf{S}}$, implies $x\left(t ; x_{0}, u\right)=U \tilde{x}\left(t ; \tilde{x}_{0}, \tilde{u}\right), y[x(t)]=\tilde{y}[\tilde{x}(t)]$, for all $t \geqslant 0$.

An expanded system $\tilde{\mathbf{S}}$ can be defined in the form $\tilde{A}=V A U+M_{A}, \tilde{B}=V B Q+N_{B}, \tilde{C}_{y}=T C_{y} U+$ $L_{C}$, where $M_{A}, N_{B}, L_{C}$ are complementary matrices of appropriate dimensions. In order to assure that the system $\mathbf{S}$ and the expanded system $\tilde{\mathbf{S}}$ satisfy the Inclusion Principle, the complementary matrices have to satisfy the following theorem.

Theorem 2. $\tilde{\mathbf{S}}$ includes the system $\mathbf{S}$ if and only if $U M_{A}^{i} V=0, U M_{A}^{i-1} N_{B} R=0, S L_{C} M_{A}^{i-1} V=0$ and $S L_{C} M_{A}^{i-1} N_{B} R=0$ for all $i=1,2, \ldots, \tilde{n}$.

A special kind of expansion-contraction scheme, called restriction, is particularly simple and suitable for the design of overlapping controllers.

Definition 3. (Restriction) Let $\tilde{\mathbf{S}}$ be an expansion of the system $\mathbf{S}$ defined by the expanded system matrices $\tilde{A}, \tilde{B}, \tilde{C}_{y}$. The system $\mathbf{S}$ is said to be a restriction of $\tilde{\mathbf{S}}$ if and only if $M_{A} V=0$, $N_{B} R=0$ and $L_{C} V=0$. 


\section{Expansions of overlapping systems}

If a system $\mathbf{S}$ can be split into three subsystems $S_{1}, S_{2}, S_{3}$ in such a way that no direct interaction between $S_{1}$ and $S_{3}$ occurs, then it admits an overlapping decomposition. From the three subsystems $S_{i}$, two overlapping subsystems $\mathbf{S}^{(1)}=\left[S_{1}, S_{2}\right], \mathbf{S}^{(2)}=\left[S_{2}, S_{3}\right]$ can be considered. More precisely, we assume that $A, B$ and $C_{y}$ present a block tridiagonal structure

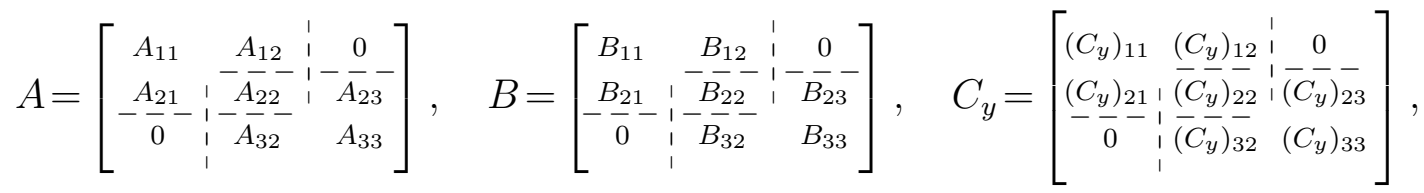

where $A_{i i}, B_{i j},\left(C_{y}\right)_{i j}$, for $i, j=1,2,3$, are $n_{i} \times n_{i}, n_{i} \times m_{j}, l_{i} \times n_{j}$ dimensional matrices, respectively. The partition of the state $x=\left(x_{1}^{T}, x_{2}^{T}, x_{3}^{T}\right)^{T}$ has components of respective dimensions $n_{1}$, $n_{2}, n_{3}$, satisfying $n_{1}+n_{2}+n_{3}=n$; the partition of $u=\left(u_{1}^{T}, u_{2}^{T}, u_{3}^{T}\right)^{T}$ has components of dimensions $m_{1}, m_{2}, m_{3}$, such that $m_{1}+m_{2}+m_{3}=m$; and $y=\left(y_{1}^{T}, y_{2}^{T}, y_{3}^{T}\right)^{T}$ has components of respective dimensions $l_{1}, l_{2}, l_{3}$, satisfying $l_{1}+l_{2}+l_{3}=l$.

Given a linear system $\mathbf{S}$, a usual choice of the expansion matrices is

$$
V=\left[\begin{array}{ccc}
I_{n_{1}} & 0 & 0 \\
0 & I_{n_{2}} & 0 \\
0 & I_{n_{2}} & 0 \\
0 & 0 & I_{n_{3}}
\end{array}\right], \quad R=\left[\begin{array}{ccc}
I_{m_{1}} & 0 & 0 \\
0 & I_{m_{2}} & 0 \\
0 & I_{m_{2}} & 0 \\
0 & 0 & I_{m_{3}}
\end{array}\right], \quad T=\left[\begin{array}{ccc}
I_{l_{1}} & 0 & 0 \\
0 & I_{l_{2}} & 0 \\
0 & I_{l_{2}} & 0 \\
0 & 0 & I_{l_{3}}
\end{array}\right]
$$

which provides the corresponding pseudoinverse contractions $U, Q$ and $S$. A first set of expanded matrices is computed in the form $\bar{A}=V A U, \bar{B}=V B Q, \bar{C}_{y}=T C_{y} U$. Then, we form an expanded system $\tilde{\mathbf{S}}$ by adding adequate complementary matrices. If the complementary matrices are chosen in the form

$$
M_{A}=\left[\begin{array}{rrrr}
0 & \frac{1}{2} A_{12} & -\frac{1}{2} A_{12} & 0 \\
0 & \frac{1}{2} A_{22} & -\frac{1}{2} A_{22} & 0 \\
0 & -\frac{1}{2} A_{22} & \frac{1}{2} A_{22} & 0 \\
0 & -\frac{1}{2} A_{32} & \frac{1}{2} A_{32} & 0
\end{array}\right], \quad N_{B}=\left[\begin{array}{rrrr}
0 & \frac{1}{2} B_{12} & -\frac{1}{2} B_{12} & 0 \\
0 & \frac{1}{2} B_{22} & -\frac{1}{2} B_{22} & 0 \\
0 & -\frac{1}{2} B_{22} & \frac{1}{2} B_{22} & 0 \\
0 & -\frac{1}{2} B_{32} & \frac{1}{2} B_{32} & 0
\end{array}\right], \quad L_{C}=\left[\begin{array}{rrrr}
0 & \frac{1}{2}\left(C_{y}\right)_{12} & -\frac{1}{2}\left(C_{y}\right)_{12} & 0 \\
0 & \frac{1}{2}\left(C_{y}\right)_{22} & -\frac{1}{2}\left(C_{y}\right)_{22} & 0 \\
0 & -\frac{1}{2}\left(C_{y}\right)_{22} & \frac{1}{2}\left(C_{y}\right)_{22} & 0 \\
0 & -\frac{1}{2}\left(C_{y}\right)_{32} & \frac{1}{2}\left(C_{y}\right)_{32} & 0
\end{array}\right],
$$

then, the system $\mathbf{S}$ is a restriction of $\tilde{\mathbf{S}}$, and the expanded system $\tilde{\mathbf{S}}$ presents an almost-decoupled structure. More specifically, the system matrices of $\tilde{\mathbf{S}}$ are given by

$$
\tilde{A}=\bar{A}+M_{A}=\left[\begin{array}{ll}
\tilde{A}_{11} & \tilde{A}_{12} \\
\tilde{A}_{21} & \tilde{A}_{22}
\end{array}\right]=\left[\begin{array}{cc:cc}
A_{11} & A_{12} & 0 & 0 \\
A_{21} & A_{22} & 0 & A_{23} \\
\hdashline A_{21} & 0 & A_{22} & A_{23} \\
0 & 0 & A_{32} & A_{33}
\end{array}\right]
$$

and similar structures have the matrices $\tilde{B}$ and $\tilde{C}_{y}$. The state, input and output vectors of the expanded system

$$
\tilde{\mathbf{S}}:\left\{\begin{array}{l}
\dot{\tilde{x}}(t)=\tilde{A} \tilde{x}(t)+\tilde{B} \tilde{u}(t) \\
\tilde{y}(t)=\tilde{C}_{y} \tilde{x}(t)
\end{array}\right.
$$

can be written in the form $\tilde{x}^{T}=\left(x_{1}^{T}, x_{2}^{T}, x_{2}^{T}, x_{3}^{T}\right), \tilde{u}^{T}=\left(u_{1}^{T}, u_{2}^{T}, u_{2}^{T}, u_{3}^{T}\right)$ and $\tilde{y}^{T}=\left(y_{1}^{T}, y_{2}^{T}, y_{2}^{T}, y_{3}^{T}\right)$.

Using the block notation given in (6), and removing the interconnection blocks, two decoupled expanded subsystems result

$$
\tilde{\mathbf{S}}_{\mathbf{D}}^{(1)}:\left\{\begin{array}{l}
\dot{\tilde{x}}_{1}(t)=\tilde{A}_{11} \tilde{x}_{1}(t)+\tilde{B}_{11} \tilde{u}_{1}(t) \\
\tilde{y}_{1}(t)=\left(\tilde{C}_{y}\right)_{11} \tilde{x}_{1}(t)
\end{array} \quad \tilde{\mathbf{S}}_{\mathbf{D}}^{(2)}:\left\{\begin{array}{l}
\dot{\tilde{x}}_{2}(t)=\tilde{A}_{22} \tilde{x}_{2}(t)+\tilde{B}_{22} \tilde{u}_{2}(t) \\
\tilde{y}_{2}(t)=\left(\tilde{C}_{y}\right)_{22} \tilde{x}_{2}(t)
\end{array}\right.\right.
$$


which define a decoupled expanded system

$$
\tilde{\mathbf{S}}_{\mathbf{D}}:\left\{\begin{array}{l}
\dot{\tilde{x}}(t)=\tilde{A}_{D} \tilde{x}(t)+\tilde{B}_{D} \tilde{u}(t) \\
\tilde{y}(t)=\left(\tilde{C}_{y}\right)_{D} \tilde{x}(t)
\end{array}\right.
$$

where $\tilde{A}_{D}=\operatorname{diag}\left\{\tilde{A}_{11}, \tilde{A}_{22}\right\}, \tilde{B}_{D}=\operatorname{diag}\left\{\tilde{B}_{11}, \tilde{B}_{22}\right\}$ and $\left(\tilde{C}_{y}\right)_{D}=\operatorname{diag}\left\{\left(\tilde{C}_{y}\right)_{11},\left(\tilde{C}_{y}\right)_{22}\right\}$.

\section{Design of overlapping controllers}

To complete the design of an overlapping controller for $\mathbf{S}$, two additional steps are required: (1) to design a decentralized controller for the expanded decoupled system $\tilde{\mathbf{S}}_{\mathbf{D}}$, and (2) to contract the decentralized expanded controller to a semi-decentralized overlapping controller for $\mathbf{S}$. The design of the decentralized controller for $\tilde{\mathbf{S}}_{\mathrm{D}}$ can be done by independently computing local controllers for $\tilde{\mathbf{S}}_{\mathbf{D}}^{(1)}$ and $\tilde{\mathbf{S}}_{\mathbf{D}}^{(2)}$.

Definition 4. (Contractibility) Suppose that $\tilde{\mathbf{S}}$ is an expansion of the system S. Then, a control law $\tilde{u}(t)=\tilde{K} \tilde{x}(t)$ for $\tilde{\mathbf{S}}$ is contractible to the control law $u(t)=K x(t)$ for $\mathbf{S}$ if there exist transformations as in (2) such that, for any initial state $x_{0} \in \mathbb{R}^{\mathbf{n}}$ and any input $u(t) \in \mathbb{R}^{\mathbf{m}}$, if $\tilde{x}_{0}=V x_{0}$ and $\tilde{u}(t)=R u(t)$ then $K x\left(t ; x_{0}, u\right)=Q \tilde{K} \tilde{x}\left(t ; V x_{0}, R u\right)$, for all $t \geqslant 0$.

Proposition 5. Suppose that $\tilde{\mathbf{S}}$ is an expansion of the system $\mathbf{S}$. Then, a control law $\tilde{u}(t)=\tilde{K} \tilde{x}(t)$ for $\tilde{\mathbf{S}}$ is contractible to the control law $u(t)=K x(t)$ for $\mathbf{S}$ if and only if $Q \tilde{K} V=K, Q \tilde{K} M_{A}^{i} V=0$, $Q \tilde{K} M_{A}^{i-1} N_{B} R=0$, for $i=1, \ldots, \tilde{n}$.

If $\tilde{K}^{(1)}$ and $\tilde{K}^{(2)}$ are local controllers for the decoupled expanded subsystems $\tilde{\mathbf{S}}_{\mathbf{D}}^{(1)}$ and $\tilde{\mathbf{S}}_{\mathbf{D}}^{(2)}$, then a block diagonal controller can be obtained in the form $\tilde{K}_{D}=\left[\begin{array}{cc}\tilde{K}^{(1)} & 0 \\ 0 & \tilde{K}^{(2)}\end{array}\right]$. This expanded controller can be contracted to an overlapping controller

$$
K_{o}=Q \tilde{K}_{D} V=\left[\begin{array}{cc:c}
K_{11} & K_{12} & 0 \\
K_{21} & K_{22} & K_{23} \\
\hdashline 0 & K_{32} & K_{33}
\end{array}\right] \text {. }
$$

\section{LQR overlapping controllers:}

To design a centralized state-feedback optimal LQR controller for the system (1), we consider the performance index

$$
J_{c}(x(t), u(t))=\int_{0}^{\infty}\left[x^{T}(t) Q^{*} x(t)+u^{T}(t) R^{*} u(t)\right] d t
$$

where $Q^{*}$ is a positive-semidefinite real symmetric matrix, and $R^{*}$ is a positive-definite real symmetric matrix. If the Riccati equation $A^{T} P+P A-P B\left(R^{*}\right)^{-1} B^{T} P+Q^{*}=0$ has a positive-definite solution $P$, then the control vector $u_{o p t}(t)=-K_{\text {opt }} x(t)$ with the gain matrix $K_{\text {opt }}=\left(R^{*}\right)^{-1} B^{T} P$ minimizes the index (11). To design an overlapping LQR controller, we start by computing local optimal LQR controllers for the expanded decoupled subsystems $\tilde{\mathbf{S}}_{\mathrm{D}}^{(1)}$ and $\tilde{\mathbf{S}}_{\mathrm{D}}^{(2)}$ (see Fig. 1). In the decoupled expanded system $\tilde{\mathbf{S}}_{\mathbf{D}}$, the gain matrix of the controller $\tilde{u}(t)=-\tilde{K}_{D} \tilde{x}(t)$ which minimizes the cost function

$$
\tilde{J}_{D}(\tilde{x}(t), \tilde{u}(t))=\int_{0}^{\infty}\left[\tilde{x}^{T}(t) \tilde{Q}_{D}^{*} \tilde{x}(t)+\tilde{u}^{T}(t) \tilde{R}_{D}^{*} \tilde{u}(t)\right] d t
$$




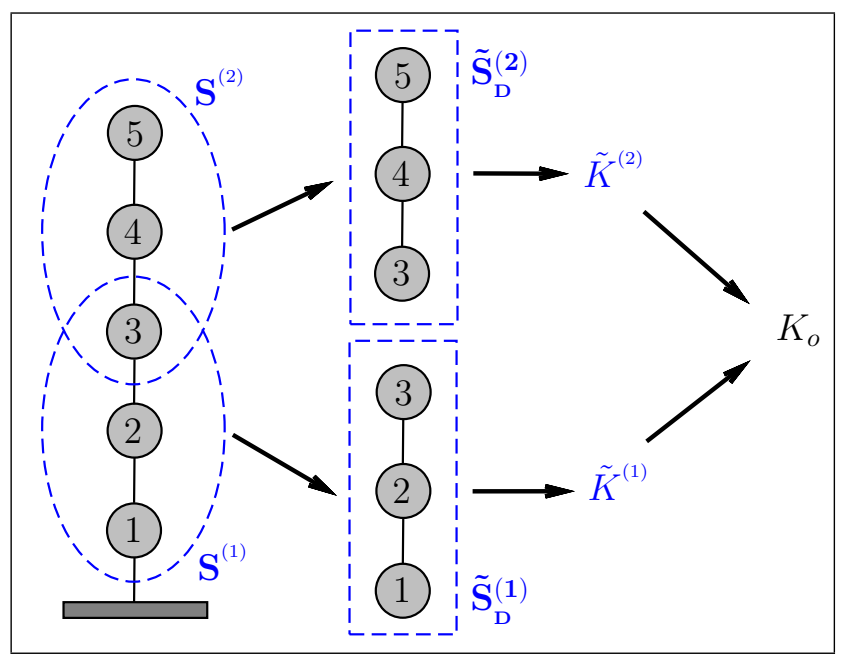

Fig. 1: Overlapping controller design for two overlapped subsystems with $\tilde{Q}_{D}^{*}=\operatorname{diag}\left\{\tilde{Q}_{1}^{*}, \tilde{Q}_{2}^{*}\right\}, \tilde{R}_{D}^{*}=\operatorname{diag}\left\{\tilde{R}_{1}^{*}, \tilde{R}_{2}^{*}\right\}$, can be written as a block diagonal gain matrix $\tilde{K}_{D}=\operatorname{diag}\left\{\tilde{K}^{(1)}, \tilde{K}^{(2)}\right\}$. Finally, the controller $\tilde{u}_{D}(t)=-\tilde{K}_{D} \tilde{x}(t)$ is contracted to an overlapping controller $u_{o}(t)=-K_{o} x(t)$ that can be implemented into the original system $\mathbf{S}$. The contracted gain matrix is computed as $K_{o}=Q \tilde{K}_{D} V$, having the block tridiagonal structure shown in (10).

Although the vast majority of theoretical results and applications of overlapping decomposition has been formulated for the simple case of two overlapping subsystems, the generalization from a simple overlapping to a multi-overlapping approach is by no means straightforward (see Fig. 3). A detailed study of the design of multi-overlapping controllers for longitudinal multi-overlapping systems following the multi-step approach can be found in [15].

\section{Five-story building model}

In this section, a simplified dynamical model for the vibrational response of a five-story building is considered (see Fig. 2). The building motion can be described by the second-order model

$$
M \ddot{q}(t)+C \dot{q}(t)+K q(t)=T_{u} u(t)+T_{w} w(t),
$$

where $M, C, K$ are, respectively, the mass, the damping and the stiffness matrices. The vector of story displacements with respect to the ground is $q(t)=\left[q_{1}(t), q_{2}(t), q_{3}(t), q_{4}(t), q_{5}(t)\right]^{T}$, with $q_{i}(t)$ representing the displacement of the $i$ th story. The vector of control forces has a similar structure, $u(t)=\left[u_{1}(t), u_{2}(t), u_{3}(t), u_{4}(t), u_{5}(t)\right]^{T}$, where $u_{i}(t)$ denotes the control force exerted by the $i$ th actuation device. $T_{u}$ is the control location matrix, $w(t)$ is the seismic ground acceleration, and $T_{w}=-M[1]_{5 \times 1}$ is the disturbance input matrix, where $[1]_{5 \times 1}$ denotes a column vector of dimension 5 with all its entries equal to 1.

The mass, damping and stiffness matrices in equation (13) are the following:

$$
M=\left[\begin{array}{ccccc}
m_{1} & 0 & 0 & 0 & 0 \\
0 & m_{2} & 0 & 0 & 0 \\
0 & 0 & m_{3} & 0 & 0 \\
0 & 0 & 0 & m_{4} & 0 \\
0 & 0 & 0 & 0 & m_{5}
\end{array}\right], C=10^{6} \times\left[\begin{array}{ccccc}
1.14 & -0.51 & 0 & 0 & 0 \\
-0.51 & 1.14 & -0.51 & 0 & 0 \\
0 & -0.51 & 1.14 & -0.51 & 0 \\
0 & 0 & -0.51 & 1.14 & -0.51 \\
0 & 0 & 0 & -0.51 & 0.63
\end{array}\right], K=\left[\begin{array}{ccccc}
k_{1}+k_{2} & -k_{2} & 0 & 0 & 0 \\
-k_{2} & k_{2}+k_{3} & -k_{3} & 0 & 0 \\
0 & -k_{3} & k_{3}+k_{4} & -k_{4} & 0 \\
0 & 0 & -k_{4} & k_{4}+k_{5} & -k_{5} \\
0 & 0 & 0 & -k_{5} & k_{5}
\end{array}\right],
$$

where $m_{j}=2.156 \times 10^{5} \mathrm{~kg}, k_{j}=1.5 \times 10^{8} \mathrm{~N} / \mathrm{m}$, for $j=1, \ldots, 5$. Ideal force-actuation devices are supposed to be placed between consecutive stories. In this case, we agree that a positive control actuation $u_{j}(t)$ will exert a positive force $u_{j}(t)$ on the $(j-1)$ th story, and a negative force $-u_{j}(t)$ on the $j$ th story. This convention is illustrated in Fig. 2. The control location matrix corresponding to the inter-story actuation scheme is $T_{u}=\left[\begin{array}{rrrrr}-1 & 1 & 0 & 0 & 0 \\ 0 & -1 & 1 & 0 & 0 \\ 0 & 0 & -1 & 1 & 0 \\ 0 & 0 & 0 & -1 & 1 \\ 0 & 0 & 0 & 0 & -1\end{array}\right]$. From the second- 


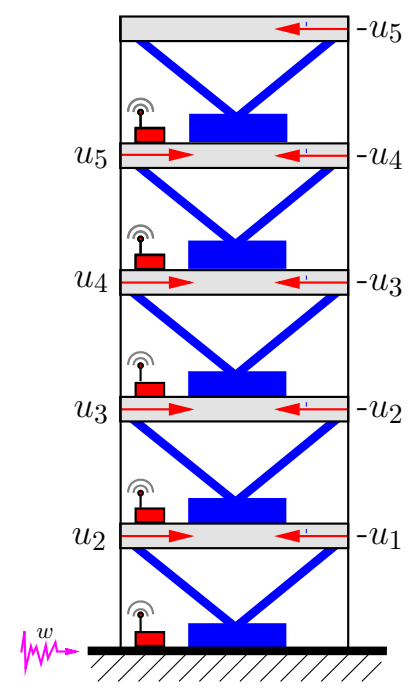

Fig. 2: Actuation scheme for a five-story building

order model (13), a first-order state-space model can be derived

$$
S_{I}: \dot{x}_{I}(t)=A_{I} x_{I}(t)+B_{I} u(t)+E_{I} w(t),
$$

by taking the state vector $x_{I}(t)=\left[\begin{array}{c}q(t) \\ \dot{q}(t)\end{array}\right]$. The state matrix in (15) and the control and distur-

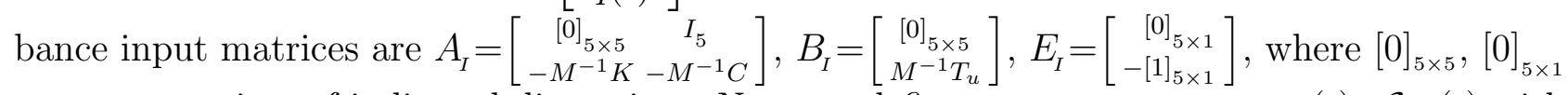
are zero-matrices of indicated dimensions. Next we define a new state vector $x(t)=\mathcal{C} x_{I}(t)$ with

$$
\mathcal{C}=\left[\begin{array}{rrrrrrrrrr}
1 & 0 & 0 & 0 & 0 & 0 & 0 & 0 & 0 & 0 \\
0 & 0 & 0 & 0 & 0 & 1 & 0 & 0 & 0 & 0 \\
-1 & 1 & 0 & 0 & 0 & 0 & 0 & 0 & 0 & 0 \\
0 & 0 & 0 & 0 & 0 & -1 & 1 & 0 & 0 & 0 \\
0 & -1 & 1 & 0 & 0 & 0 & 0 & 0 & 0 & 0 \\
0 & 0 & 0 & 0 & 0 & 0 & -1 & 1 & 0 & 0 \\
0 & 0 & -1 & 1 & 0 & 0 & 0 & 0 & 0 & 0 \\
0 & 0 & 0 & 0 & 0 & 0 & 0 & -1 & 1 & 0 \\
0 & 0 & 0 & -1 & 1 & 0 & 0 & 0 & 0 & 0 \\
0 & 0 & 0 & 0 & 0 & 0 & 0 & 0 & -1 & 1
\end{array}\right]
$$

The new state $x(t)=\left[x_{1}, \cdots, x_{10}\right]$ groups together the inter-story drifts and inter-story velocities in increasing order, that is,

$$
\begin{aligned}
x_{1}(t) & =q_{1}(t), \quad x_{2}(t)=\dot{q}_{1}(t), \\
x_{2 j-1}(t) & =q_{j}(t)-q_{j-1}(t), \text { for } j=2,3,4, \\
x_{2 j}(t) & =\dot{q}_{j}(t)-\dot{q}_{j-1}(t), \text { for } j=2,3,4, \\
x_{9}(t) & =q_{5}(t), \quad x_{10}(t)=\dot{q}_{5}(t) .
\end{aligned}
$$

The new state-space model is $\dot{x}(t)=A x(t)+B u(t)+E w(t)$, with $A=\mathcal{C} A_{I} \mathcal{C}^{-1}, B=\mathcal{C} B_{I}, E=\mathcal{C} E_{I}$. For the particular values of the building parameters, we obtain the state matrix

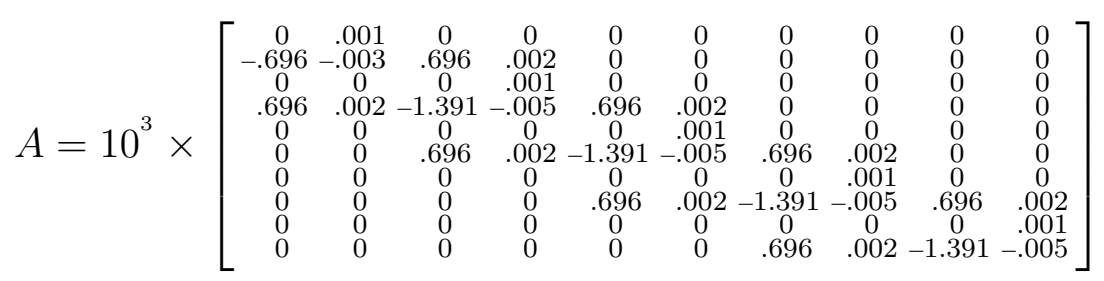


and the disturbance and control input matrices given by

$$
E=[0,-1,0,0,0,0,0,0,0,0]^{T}, \quad B=10^{-5} \times\left[\begin{array}{ccccc}
0 & 0 & 0 & 0 & 0 \\
-0.464 & 0.464 & 0 & 0 & 0 \\
0 & 0 & 0 & 0 & 0 \\
0.464 & -0.928 & 0 & 0 & 0 \\
0 & 0 & 0 & 0 & 0 \\
0 & 0.464 & -0.928 & 0.464 & 0 \\
0 & 0 & 0 & 0 \\
0 & 0 & 0.464 & -0.928 & 0 \\
0 & 0 & 0 & 0 & 0 \\
0 & 0 & 0 & 0.464 & -0.923
\end{array}\right] .
$$

\section{Controllers design}

In this section, several controllers are designed for this five-story building model. More precisely, three kinds of controllers are computed: (i) a centralized controller, (ii) a semi-decentralized two-overlapping controller, and (iii) a semi-decentralized multi-overlapping controller.

\section{Centralized controller:}

To compute a centralized optimal LQR controller, we consider the quadratic index $J_{c}$ described in (11) defined by the weighting matrices $Q^{*}=\operatorname{diag}[1,0,1,0,1,0,1,0,1,0]$ and $R^{*}=10^{-16} I_{5}$. The gain matrix of the optimal LQR controller computed with the system matrices $A, B$ given in (18), (19) is

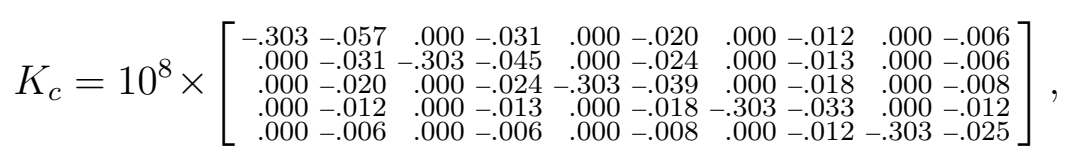

with an optimal cost $\left[J_{c}\right]_{o p t}=0.3749$. Note that the full-state is needed to compute the control vector $u_{c}=-K_{c} x(t)$ and, consequently, a full-range communication system must be used to implement the obtained centralized controller.

\section{Two-overlapping controller:}

In this subsection, we consider the overlapping decomposition depicted in Fig. 1 and we use the previous ideas to design a semi-decentralized two-overlapping controller. The considered overlapping decomposition consists of two systems $\mathbf{S}^{(1)}=[1,2,3], \mathbf{S}^{(2)}=[3,4,5]$ which overlap in the third story. The dimensions of the state partition are $n_{1}=4, n_{2}=2, n_{3}=4$; for the control partition, we have $m_{1}=2, m_{2}=1, m_{3}=2$. The values of $n_{j}, m_{j}$ define the expansion matrices $V=\left[\begin{array}{ccc}I_{4} & 0 & 0 \\ 0 & I_{2} & 0 \\ 0 & I_{2} & 0 \\ 0 & 0 & I_{4}\end{array}\right], R=\left[\begin{array}{ccc}I_{2} & 0 & 0 \\ 0 & I_{1} & 0 \\ 0 & I_{1} & 0 \\ 0 & 0 & I_{2}\end{array}\right]$. After performing the decoupled decomposition, we obtain decoupled expanded systems $\tilde{\mathbf{S}}_{\mathbf{D}}^{(1)}$ and $\tilde{\mathbf{S}}_{\mathbf{D}}^{(2)}$ together with the corresponding quadratic indexes $\tilde{J}_{D}^{(1)}$, $\tilde{J}_{D}^{(2)}$. Then, a contraction process for the obtained local gain matrices $\tilde{K}^{(1)}$ and $\tilde{K}^{(2)}$ produces a contracted gain matrix

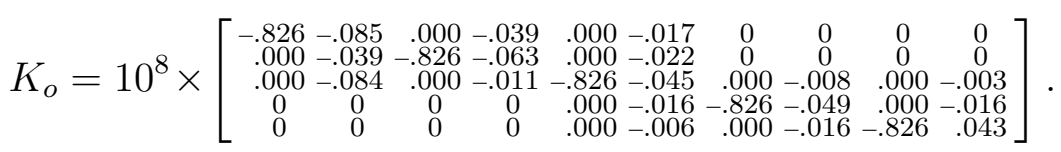

Note that, due to the particular structure of the overlapping control matrix (21), a local controller with wireless communications system would only need to cover a range of half building to compute the control vector $u_{o}=-K_{o} x(t)$. The quadratic index value $J_{c}$ corresponding to the overlapping controllers is $\left[J_{c}\right]_{K_{o}}=0.4430$.

\section{Multi-overlapping controller:}

In this subsection, the previous ideas are applied to the design of semi-decentralized multioverlapping controllers for the multi-overlapping decomposition depicted in Fig. 3. 


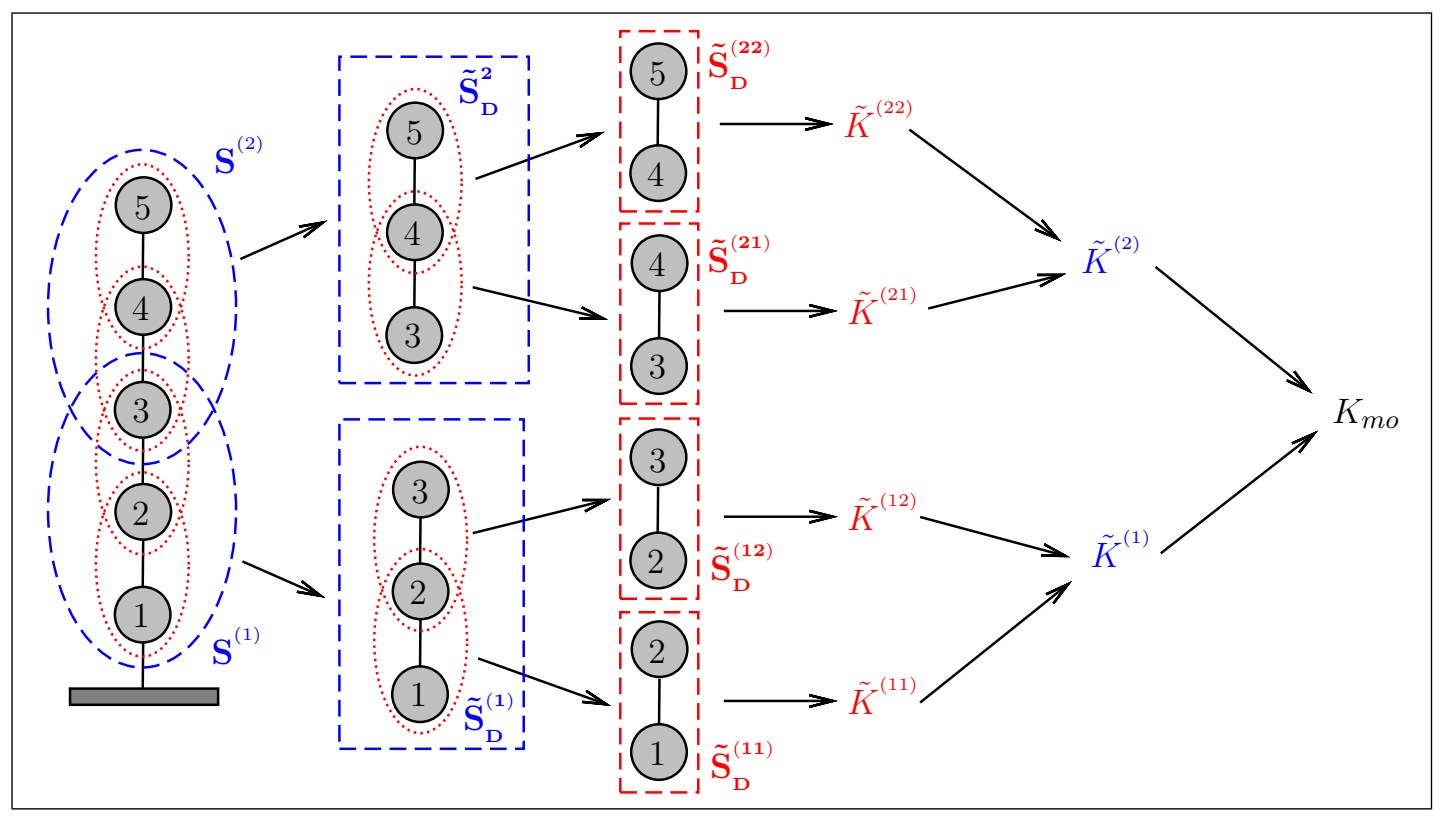

Fig. 3: Multi-overlapping expansion-contraction process

Following a similar process, the first step is to obtain two decoupled expanded decompositions $\tilde{\mathbf{S}}_{\mathbf{D}}=\left\{\tilde{\mathbf{S}}_{\mathbf{D}}^{(1)}, \tilde{\mathbf{S}}_{\mathbf{D}}^{(2)}\right\}$. Now, we observe that each expanded subsystem $\tilde{\mathbf{S}}_{\mathbf{D}}^{(1)}$ and $\tilde{\mathbf{S}}_{\mathbf{D}}^{(2)}$ admits a new overlapping decomposition and we define a second set of expansion matrices $V=\left[\begin{array}{ccc}I_{2} & 0 & 0 \\ 0 & I_{2} & 0 \\ 0 & I_{2} & 0 \\ 0 & 0 & I_{2}\end{array}\right]$, $R=\left[\begin{array}{ccc}I_{1} & 0 & 0 \\ 0 & I_{1} & 0 \\ 0 & I_{1} & 0 \\ 0 & 0 & I_{1}\end{array}\right]$ to obtain the decoupled expansions $\tilde{\mathbf{S}}_{\mathbf{D}}^{(1)}=\left\{\tilde{\mathbf{S}}_{\mathbf{D}}^{(11)}, \tilde{\mathbf{S}}_{\mathbf{D}}^{(12)}\right\}, \tilde{\mathbf{S}}_{\mathbf{D}}^{(2)}=\left\{\tilde{\mathbf{S}}_{\mathbf{D}}^{(21)}, \tilde{\mathbf{S}}_{\mathbf{D}}^{(22)}\right\}$. The expanded LQR controllers for the decoupled subsystems $\tilde{\mathbf{S}}_{\mathbf{D}}^{(i j)}$ are

$\tilde{K}^{(11)}=10^{8} \times\left[\begin{array}{rrrr}-.8264 & -.0730 & -0000 & -.0255 \\ .0000 & -.0255 & -.8264 & -.0475\end{array}\right], \quad \tilde{K}^{(12)}=\tilde{K}^{(21)}=\tilde{K}^{(22)}=10^{8} \times\left[\begin{array}{rrrr}-.8264 & -.0416 & .0000 & -.0122 \\ .0000 & -.0122 & -.8264 & -.0416\end{array}\right]$.

After two contraction steps, the following multi-overlapping controller results

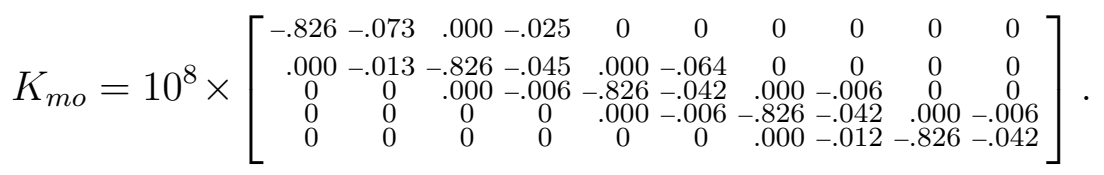

It is worth to be highlighted that only two four-dimensional LQR problems have been actually solved in the design of the ten-dimensional multi-overlapping controller. Moreover, due to the block tridiagonal structure of the multi-overlapping gain matrix (23), when the control vector $u_{m o}=-K_{m o} x(t)$ is computed, a transmission range of only one story is required. The value of the quadratic index $J_{c}$ corresponding to the multi-overlapping controller is $\left[J_{c}\right]_{K_{m o}}=0.4881$.

Remark. The relatively higher values associated to the two-overlapping and multi-overlapping controllers might be seen in the light of its remarkable features: reduced information exchange, short-range transmission requirements, and computational efficiency.

\section{Numerical simulations}

In this section, a set of graphic simulations related to the maximum absolute inter-story drifts and maximum control forces are conducted. Ground acceleration of El Centro earthquake has 


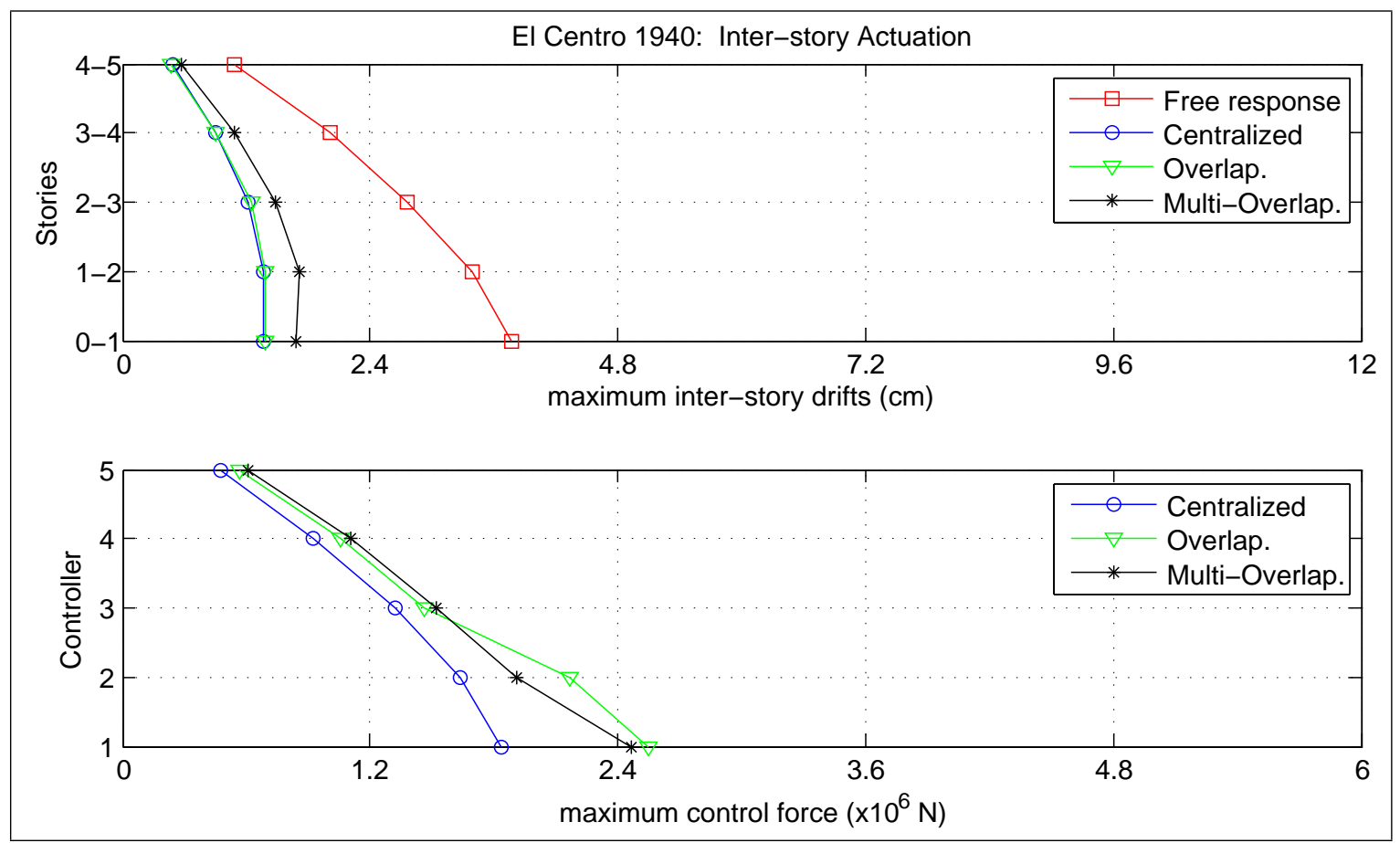

Fig. 4: Maximum inter-story drifts and control efforts

been taken as seismic disturbance. Figure 4 displays the corresponding simulation outputs, which represent the maximum absolute inter-story drifts together with the associated maximum absolute control efforts obtained for the controlled building with: (i) centralized controller (blue circles), (ii) two-overlapping controller (green triangles) (iii) multi-overlapping controller (black asterisks). The maximum absolute inter-story drifts of the uncontrolled building response (red squares) are also included as reference. The simulation results show that the two-overlapping and the multi-overlapping controllers achieve levels of performance similar to those obtained by the centralized counterpart.

\section{Conclusions}

Current trends in structural vibration control consider complex control configurations distributed over large structures. For tall buildings under seismic excitation, the design of multioverlapping controllers via the Inclusion Principle has proved to be a specially suitable choice.

\section{Acknowledgments}

This work was partially supported by the Spanish Ministry of Economy and Competitiveness under Grants DPI2011-27567-C02-02 and DPI2008-06564-C02-02.

\section{References}

[1] T.T. Soong and M.C. Constantinou. Passive and Active Structure Vibration Control in Civil Engineering. Springer, New York, 1994.

[2] B.F. Spencer and S. Nagarajaiah. State of the art of structural control. Journal of Structural Engineering, 129(7):845-856, July 2003. 
[3] G.J. Hiemenz, Y.T. Choi, and N.M. Wereley. Seismic control of civil structures utilizing semiactive MR braces. Computer-Aided Civil and Infrastructure Engineering, 18:31-44, 2003.

[4] T. Kobori. Past, present and future in seismic response control in civil engineering structures. In Proceedings of the 3rd. World Conference on Structural Control, New York, 2003.

[5] N. Kurata, T. Kobori, and N. Koshika. Performance-based design with semiactive control technique. In Earthquake Engineering Structural Dynamics, volume 31, pages 445-458, 2002.

[6] A. Nishitani and Y. Inoue. Overview of the application of active/semiactive control to building structures in Japan. Earthquake Engineering and Structural Dynamics, 30:15651574, 2001.

[7] A. Preumont and K. Seto. Active Control of Structures. Wiley, United Kingdom, 2008.

[8] J.N. Yang and A.K. Agrawal. Semi-active hybrid control systems for nonlinear buildings against near-field earthquakes. Engineering Structures, 24(3):271-280, 2002.

[9] D.D. Šiljak. Decentralized Control of Complex Systems. Academic Press, New York, USA, 1991.

[10] L. Bakule, J. Rodellar, and J. M. Rossell. Structure of expansion-contraction matrices in the inclusion principle for dynamic systems. SIAM Journal on Matrix Analysis and Applications, 21(4):1136-1155, 2000.

[11] L. Bakule, J. Rodellar, and J. M. Rossell. Generalized selection of complementary matrices in the inclusion principle. IEEE Transactions on Automatic Control, 45(6):1237-1243, 2000 .

[12] K.H. Law, J.P. Lynch, and Y. Wang. Decentralized control strategies with wireless sensing and actuation. In Proceedings of 29 NSF CMMI Engineering Research and Innovation Conference, Honolulu, Hawaii, 2009.

[13] J. Lynch, Y. Wang, R. Swartz, K. Lu, and C. Loh. Implementation of a closed-loop structural control system using wireless sensor networks. Structural Control and Health Monotoring, 15(4):518-539, 2008.

[14] Y. Wang, R.A. Swartz, J.P. Lynch, K.H. Law, K. Lu, and C. Loh. Decentralized real-time velocity feedback control of structures using wireless sensors. In Proceedings of the 4th International Conference on Earthquake Engineering, Taipei, Taiwan, 2006.

[15] F. Palacios-Quiñonero, J. Rodellar, and J. M. Rossell. Sequential design of multioverlapping controllers for longitudinal multi-overlapping systems. Journal of Applied Mathematics and Computation, 217(3):1170-1183, 2010.

[16] J. M. Rossell, F. Palacios-Quiñonero, and J. Rodellar. Semi-decentralized output feedback $H_{\infty}$ control strategy for large building structures. In Proceedings of the 5th. World Conference on Structural Control and Monitoring, Shinjuku,Tokio, July 12-14 2010. 